\title{
Miliary tuberculosis occurred after immunosuppressive drug in PNH patient with completely cured tuberculosis; a case report
}

\author{
Jihyun Lee', Soojung Gong ${ }^{1 *}$, Byounghoon Lee', Soyoung Lee', Jungae Lee ${ }^{2}$ and Naeyu Kim²
}

\begin{abstract}
Paroxysmal nocturnal hemoglobinuria (PNH) is a clonal disorder that presents with hemolytic anemia, marrow failure and thrombophilia. During acute attacks, corticosteroid can alleviate the hemolytic paroxysm, but the prolonged administration induces serious toxicity including immunosuppression. So American thoracic society (ATS) for tuberculosis (TB) recommends prophylactic anti-TB medication in patients with a long-term steroid therapy. However, in the patient who was treated for active TB in the past, there are no guidelines of the test for determining patients who have latent TB infection (LTBI) and no recommendations of TB prophylaxis if there is no evidence of reactivation at present. A 40-year-old male patient presented with fever and aggravated weakness for a week. He was diagnosed with PNH a month ago and took corticosteroid for 3 weeks. In the past, he was diagnosed with pulmonary TB and completely cured after treatment. According to guideline, he was not indicated with TB prophylaxis. However, he caught miliary TB, progressed to acute respiratory distress syndrome. We experience this embarrassing case, and emphasize the need to investigate multicentral TB prevalence and to make the guidelines of anti-TB medication in subgroups of hematologic diseases including PNH.
\end{abstract}

Keywords: Paroxysmal nocturnal hemoglobinuria, Miliary tuberculosis (TB), Tuberculosis (TB) prophylaxis

\section{Introduction}

Paroxysmal nocturnal hemoglobinuria $(\mathrm{PNH})$ is a rare acquired clonal hematopoietic stem cell disorder that presents with hemolytic anemia, venous thrombosis, deficient hematopoiesis and chronic renal disease [1]. It is a serious chronic disease with high morbidity and mortality. Hematopoietic stem cell transplantation is only a curative treatment. The treatment is personalized and directed towards the specific complications. Sometimes, PNH patients have acute attacks. Corticosteroid and androgen can be lessen a hemolytic paroxysm and improve hemoglobin levels in about $60 \%$ of PNH patients. However, the required doses of corticosteroid are high, and a long-term continuous administration on a daily basis is difficult due to its toxicities [2,3]. Owing to immunosuppressive effect of corticosteroid, American thoracic society (ATS) for tuberculosis (TB) recommends anti-TB

\footnotetext{
* Correspondence: gsj1117@eulji.ac.kr

${ }^{1}$ Department of Internal Medicine, Eulji University College of Medicine, Eulji Medical Center, 14 Hangeulbiseok-gil, Nowon-gu, Seoul 139-872, South Korea Full list of author information is available at the end of the article
}

medication in patients used prednisone more than $15 \mathrm{mg} /$ day over a month [4]. Nevertheless, these guidelines show only the treatment principles, in clinical practice, there are some problems to decide the test or management in special groups including hematologic diseases. In addition, the prevalence of miliary TB in malignant disease is 3 times higher than in general population and its mortality rate is about $25-30 \%$ in adults [5].

We experienced a PNH patient with acute attack. He received corticosteroid for 3 weeks to control hemolysis. In the past, he was diagnosed with pulmonary $\mathrm{TB}$ and we investigated LTBI (latent TB infection). He was not indicated with anti-TB medication [6], but after immunosuppressive drug for 3 weeks, he suffered from miliary TB. So we report this difficult and embarrassing case with literature review of $\mathrm{TB}$ associated with hematologic disease.

\section{Case report}

A 40-year-old Asian male patient presented with fever and more aggravated generalized weakness for 1 week. A

\section{Biomed Central}


month ago, he complained of hematuria, fever and generalized weakness. He was diagnosed with $\mathrm{PNH}$ associated with another bone marrow disorder (aplastic anemia). For control of acute hemolysis, red cells transfusion and immunosuppressive drugs (corticosteroid and danazol) were prescribed and he was discharged after stabilized. At that time, his chest X-ray showed old pulmonary calcified nodules in both lung field (Figure 1). He was diagnosed with pulmonary $\mathrm{TB}$ about 21 years ago and he was completely cured after anti-TB medication (Isoniazid, Ethambutol, Rifampicin, Pyrazinamide) for 6 months. On readmission, vital signs were followed; BP $140 / 80 \mathrm{mmHg}$, pulse rate 76 beats/min, respiration rate 20 times/min, and body temperature $39.1^{\circ} \mathrm{C}$. Jaundice was observed in both sclera, and breath sounds were decreased in both lung field. The laboratory findings revealed white blood cells, 720/uL (segment neutrophil 75.6\%; lymphocytes 15.7\%); hemoglobin, $9.6 \mathrm{~g} / \mathrm{dL}$; and platelets, 55,000/uL. AST/ALT, total bilirubin and LDH were $167 / 312 \mathrm{IU} / \mathrm{L}, 3.9 \mathrm{mg} / \mathrm{dL}$ and 2,543 IU/L respectively. Blood urea nitrogen and serum creatinine were $20.3 \mathrm{mg} / \mathrm{dL}$ and $1.4 \mathrm{mg} / \mathrm{dL}$. In chest radiographs, the miliary nodules were observed in both lung field (Figure 2A). Chest CT scan showed miliary nodulation and patch underlying perinodular ground glass appearance in both lung field, suggestive of miliary TB (Figure 2B). TB PCR was positive. After 3 weeks of

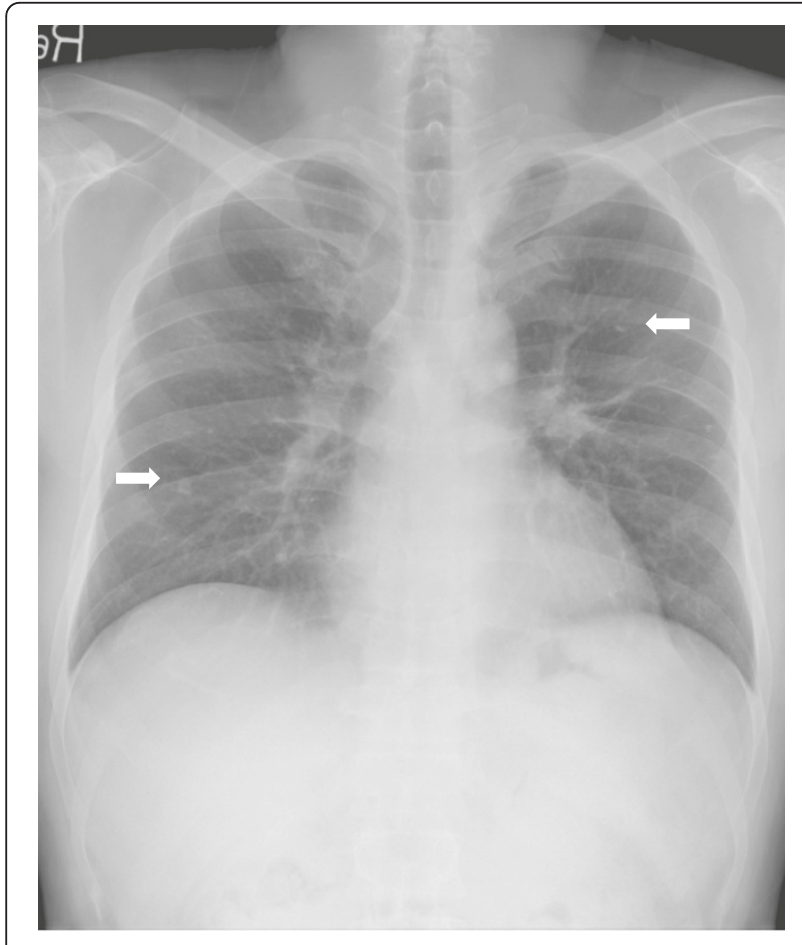

Figure 1 In chest radiographs, the suspicious old tuberculous calcified nodule was seen (white arrow). immunosuppressive drugs, he was diagnosed to miliary TB. Due to elevated liver enzyme, Ethambutol/Moxifloxacin/Cycloserine were selected. To expect the rise of drug effects, supportive care such as G-CSF administration, blood transfusion and nutritional support were done. After 5 days of the anti-TB medications, patch density in chest X-ray was progressed and he complained of severe dyspnea, nausea, vomiting and aggravated weakness (Figure 3). We considered ventilator care, but the patient was tolerable at the $10 \mathrm{~L}$ of oxygen mask apply. We added $1^{\text {st }}$ line anti-TB drugs (Isoniazid, Rifampicin) stage by stage. After continuous intensive care for 3 weeks, dyspnea was getting better and he needed only $2 \mathrm{~L}$ of oxygen apply. The chest X-ray has got better. He was improved and the resistance strain was not seen in 8 weeks after culture. He had taken $1^{\text {st }}$ line anti-TB drugs (Isoniazid, Ethambutol, Rifampicin) and warfarin in outpatient of hospital, and nowadays he finished anti-TB drugs except warfarin (Figure 4).

\section{Discussion}

In paroxysmal nocturnal hemoglobinuria $(\mathrm{PNH})$, the absence of the glycosyl phosphatidylinositol (GPI)-anchored proteins induces hemolytic anemia, thrombosis and smooth muscle dystonias. PNH is a chronic condition and its treatment includes 1) allogeneic bone marrow transplantation, 2) eculizumab, a humanized monoclonal antibody against the terminal complement protein $\mathrm{C} 5[7,8]$ and 3) supportive care such as transfusion, iron and folic acid supplementation and prophylactic anti-coagulation. However, sometimes patients have acute attacks. Corticosteroid can alleviate a hemolytic paroxysm and improve hemoglobin levels in about $60 \%$ of $\mathrm{PNH}$ patients. But a long-term continuous administration of high dose corticosteroid is troubled with its toxicity [2]. Due to immunosuppression of corticosteroid, CDC recommends prophylactic anti-TB medication in patients with more than $15 \mathrm{mg} /$ day prednisone over 1 month [4].

Worldwide, for the elimination of TB, identification and treatment of latent tuberculosis infection (LTBI) are essential [9-12]. LTBI is a condition in which a person is infected with dormant Mycobacterium tuberculosis organisms, but does not currently have active TB disease [13]. According to ATS for TB, following patients with LTBI should be considered for anti-TB medication; HIV infection, organ transplantation related to immunesuppressant therapy, recent TB infection within 2 years, TNF inhibitors. In cellular immunity of TB, lymphocyte is a major part of immune activity $[4,14]$. In patients with hematologic disease, T-cell immunodeficiency was induced by hematologic disease itself, chemotherapy, corticosteroid and other underlying diseases [15]. Hahn et al. reported that the prevalence of active $\mathrm{TB}$ in hematologic disorders was $0.8 \%$ in total, with $21.4 \%$ in 


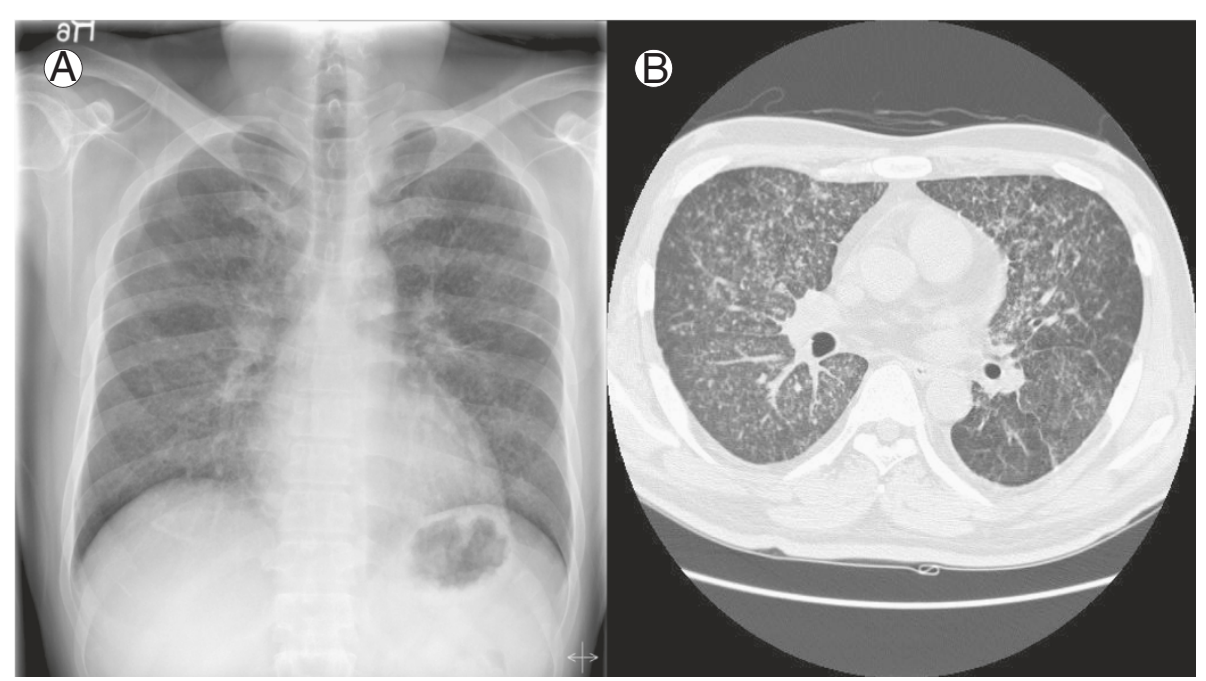

Figure 2 (A) In chest radiographs, the miliary nodules were observed in both lung field. (B) Chest CT scan showed miliary nodulation and patch underlying perinodular ground glass appearance in both lung field, suggestive of miliary TB.

myelofibrosis, $20 \%$ in $\mathrm{PNH}$ and $7.9 \%$ in myelodysplastic syndrome in Korea. Although in this report, the prevalence of TB in the hematologic disorder was not significantly different compared with control group, the incidence of TB in PNH was very high [16]. There are many controversies in the incidence of $\mathrm{TB}$ in

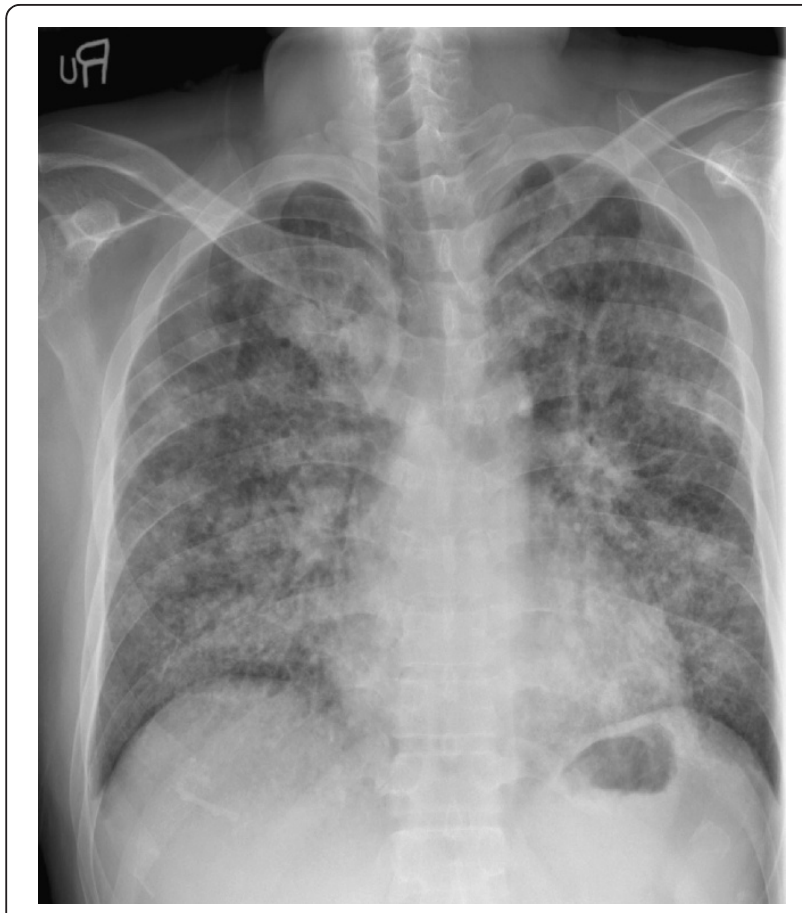

Figure 3 Patch density in chest X-ray was progressed, suggestive of acute respiratory distress syndrome. immunosuppressed patients. In clinical practice, considering risk and an incidence, the diagnosis and treatment of LTBI should be determined. However, there is a problem in determination of prophylactic anti-TB medication, that is to say limitation of the diagnostic methods for LTBI in immunosuppressed patients.

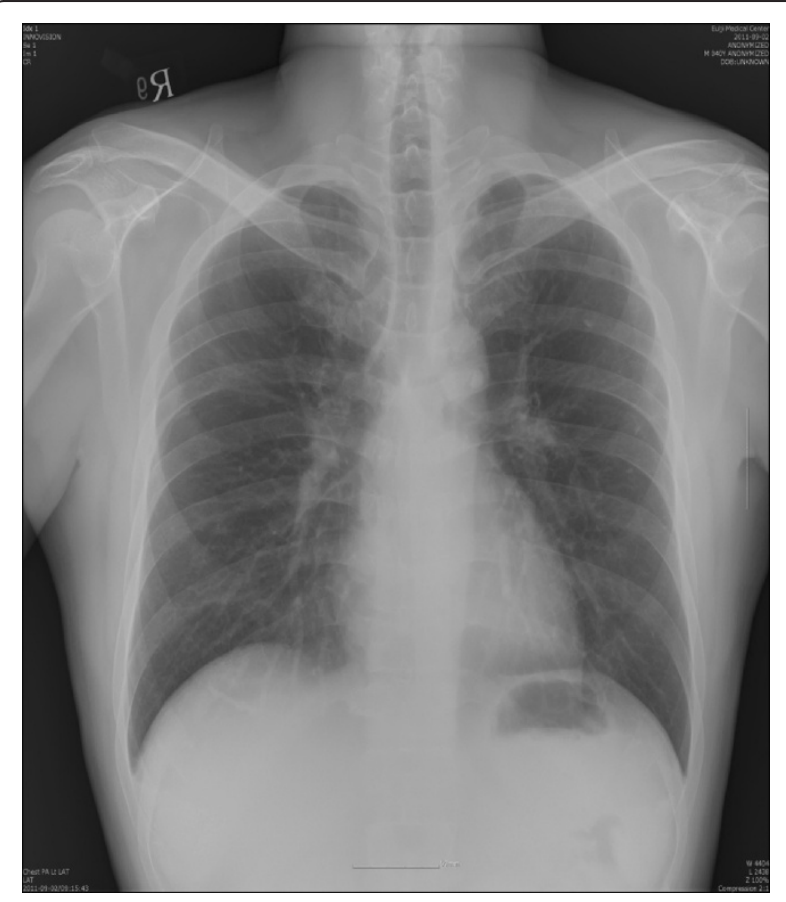

Figure 4 After anti-tuberculosis medication, chest X-ray showed improvement. 
American Thoracic Society (ATS) proposed targeted tuberculin skin test (TST) as a diagnostic tool for LTBI in high-risk group and in the U.S. diagnostic guidelines of 2000, an immunologic test was described. QunatiFERON-TB Gold (QFT-G) is an interferon-gamma releasing assay (IGRA) and it was approved by the FDA for the diagnosis of TB in 2005 and replaced TST $[4,17]$. In Korea with high prevalence of TB and high false positivity of TST due to BCG vaccination, IGRA replace TST. But IGRA has a limitation in differentiation of active TB from LTBI, and when patient was treated with LTBI or active TB in the past, it can't determine whether or not new infection is developed. Also in patients with immunosuppression and complete recovery from $\mathrm{TB}$ at the same time, there is no guideline.

On the other hand, miliary $\mathrm{TB}$ accounts for about $1 \%$ of all cases of $\mathrm{TB}$ and the mortality related to miliary TB is about $25-30 \%$ in adults [5]. Miliary TB may infect any number of organs, including the lungs, liver and spleen. Hematologic abnormalities are frequently described and pancytopenia is profound in hematolgic diseases. In malignant diseases, the incidence of miliary TB is about $3 \%$ and it rarely reported in hematologic diseases $[18,19]$.

In our patient, before the administration of immunosuppressive drugs for control of acute hemolysis, we wanted to perform the test for detection of LTBI according to CDC for TB. However, since our patient did not have clinical and radiological evidence of active $\mathrm{TB}$ and he was completely cured TB in the past, he was not indicated to test and prophylactic anti-TB medication. In addition, because anti-TB medication frequently induces hepatic dysfunction, poor oral intake, nausea and vomiting, the administration prophylactic anti-TB medication is very troubled in seriously ill patient out of guideline. However, he contracted miliary $\mathrm{TB}$ and we were in confusion. The treatment for TB was not easy because he complained of nausea, vomiting and poor oral intake and his laboratory findings showed severe pancytopenia and liver dysfunction. Meanwhile, fever is the sign that needs differential diagnosis. He had fever at diagnosis of $\mathrm{PNH}$ due to hemolysis and he was checked chest X-ray regularly (weekly). But, miliary $\mathrm{TB}$ was developed in one week. So in patients with fever and immunosuppressive drugs, inspection and chest X-ray at intervals of 2 or 3 days are needed.

In conclusion, except some advanced country, TB is endemic disease in worldwide yet. Also, there are differences of $\mathrm{TB}$ prevalence between the subgroups of hematologic diseases. So we emphasize the need to investigate multicentral $\mathrm{TB}$ prevalence and to make an urgent decision of details of the guidelines for
anti-TB medication in patients with subgroups of hematologic diseases including PNH.

\section{Consent}

Written informed consent was obtained from the patient for publication of this case report and any accompanying images. A copy of the written consent is available for review by the Editor-in-Chief of Annals of clinical microbiology and antimicrobials.

\section{Competing interests}

The authors declare that they have no competing interests.

\section{Authors' contributions}

$J$ and SG participated in the concept and design of the manuscript. BL provided pulmonologic concept of this manuscript. All authors read and approved the final manuscript.

\section{Author details}

${ }^{1}$ Department of Internal Medicine, Eulji University College of Medicine, Eulji Medical Center, 14 Hangeulbiseok-gil, Nowon-gu, Seoul 139-872, South Korea. ${ }^{2}$ Department of Internal Medicine, Eulji University College of Medicine, Eulji Medical Center, Dunsan-dong, Seo-gu, Daejeon 1306, South Korea.

Received: 5 January 2012 Accepted: 17 March 2012

Published: 3 May 2012

\section{References}

1. Rachidi S, Musallam KM, Taher AT: A closer look at paroxysmal nocturnal hemoglobinuria. Eur J Intern Med 2010, 21:260-267.

2. Brodsky RA: How I treat paroxysmal nocturnal hemoglobinuria. Blood 2009, 113:6522-6527.

3. Parker CJ, Brodsky RA, Levine JE: Treatment versus transplant for challenging hematologic disorders. Biol Blood Marrow Transplant 2009, 15:72-78.

4. Centers for Disease Control and Prevention (CDC): Targeted tuberculin testing and treatment of latent tuberculosis infection. American Thoracic Society. MMWR 2000, 49:1-51.

5. Sharma SK, Mohan A, Sharma A, Mitra DK: Miliary tuberculosis: new insights into an old disease. Lancet Infect Dis 2005, 5:415-430.

6. Falk A, Fuchs GF: Prophylaxis with isoniazid in inactive tuberculosis. A Veterans Administration Cooperative Study XII. Chest 1978, 73:44-48.

7. Kim JS, Lee JW, Kim BK, Lee JH, Chung J: The use of the complement inhibitor eculizumab (Soliris(R)) for treating Korean patients with paroxysmal nocturnal hemoglobinuria. Korean J Hematol 2010, 45:269-274.

8. Hillmen P, Young NS, Schubert J, Brodsky RA, Socie G, Muus P, Roth A, Szer $J$, Elebute MO, Nakamura R, et al: The complement inhibitor eculizumab in paroxysmal nocturnal hemoglobinuria. N Engl J Med 2006, 355:1233-1243.

9. Shim TS, Koh WJ, Yim JJ, Lew WJ: Treatment of Latent Tuberculosis Infection in Korea. Tubercul Respir Dis 2008, 65:79-90.

10. Lobue P, Menzies D: Treatment of latent tuberculosis infection: an update. Respirology 2010, 15:603-622.

11. World Health Organization: Global Tuberculosis Control: A Short Update to the 2009 Report; 2009.

12. Shim TS: Comparison of guidelines for the management of tuberculosis: Korea, United States, and World Health Organization. J Korean Med Sci 2006, 49:781-789.

13. Jasmer RM, Nahid P, Hopewell PC: Clinical practice. Latent tuberculosis infection. N Engl J Med 2002, 347:1860-1866.

14. Bhatt K, Salgame P: Host innate immune response to Mycobacterium tuberculosis. J Clin Immunol 2007, 27:347-362.

15. Silva FA, Matos JO, de QMFC, Nucci M: Risk factors for and attributable mortality from tuberculosis in patients with hematologic malignances. Haematologica 2005, 90:1110-1115.

16. Hahn JS, Lee YC, Kim YK, Lee SJ, Ko YW, Chang J, Lee WY: Clinical study on tuberculosis in patients with hematologic disease. Korean J Hematol 1990, 25:161-169. 
17. World Health Organization (WHO): WHO report 2010 Global tuberculosis control. WHO report 2010 2010:1-218.

18. Sugino K, Gocho K, Ota H, Kobayashi M, Sano G, Isobe K, Takai Y, Izumi H, Kuraishi Y, Shibuya K, Homma S: Miliary tuberculosis associated with chronic neutrophilic leukemia. Intern Med 2009, 48:1283-1287.

19. Neonakis IK, Alexandrakis MG, Gitti Z, Tsirakis G, Krambovitis E, Spandidos DA: Miliary tuberculosis with no pulmonary involvement in myelodysplastic syndromes: a curable, yet rarely diagnosed, disease: case report and review of the literature. Ann Clin Microbiol Antimicrob $2008,7: 8$.

doi:10.1186/1476-0711-11-12

Cite this article as: Lee et al:: Miliary tuberculosis occurred after immunosuppressive drug in PNH patient with completely cured tuberculosis; a case report. Annals of Clinical Microbiology and Antimicrobials 2012 11:12

\section{Submit your next manuscript to BioMed Central and take full advantage of:}

- Convenient online submission

- Thorough peer review

- No space constraints or color figure charges

- Immediate publication on acceptance

- Inclusion in PubMed, CAS, Scopus and Google Scholar

- Research which is freely available for redistribution 